\title{
Down-regulated long non-coding RNA H19 inhibits carcinogenesis of renal cell carcinoma
}

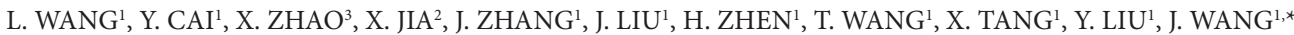

${ }^{1}$ Department Of Urology, Zhengzhou Central Hospital Affiliated To Zhengzhou University, Zheng Zhou, 450007, China; ${ }^{2}$ Department Of Breat surgery, Zhengzhou Central Hospital Affiliated To Zhengzhou University, Zheng Zhou, 450007, China; ${ }^{3}$ Department Of Urology, The Second Affiliated Hospital Of Zhengzhou University, Zheng zhou, 450007, China

*Correspondence: jizhengwang81@163.com

Received August 4, 2014/ Accepted November 13, 2014

Long non-coding RNAs (lncRNAs) have been shown to have important regulatory roles in cancer biology. LncRNA H19 has been recently shown to be upregulated and play important roles in several cancers such as breast cancer, bladder cancer, and gastric cancer. However, the role of H19 in clear cell renal carcinoma (ccRCC) remains largely unknown.

The expression levels of lncRNA H19 in ccRCC tissues and renal cancer cell lines were evaluated by quantitative Real-time PCR (qRT-PCR). And its association with overall survival of patients was analyzed by statistical analysis. Small interfering RNA (siRNA) was used to suppress H19 expression in renal cancer cell lines. In vitro assays were performed to further explore its role in tumor progression.

The relative level of $\mathrm{H} 19$ was significantly higher in ccRCC compared to the adjacent normal renal tissues. And higher expression of $\mathrm{H} 19$ was found in renal cancer cells compared to the nonmalignant renal cells HK-2. Furthermore, The ccRCC patients with higher H19 expression had more advanced clinical stage and poorer prognosis than those with lower expression, Kaplan-Meier analysis revealed that patients with higher H19 expression had a poorer overall survival and H19 expression could be an independent prognostic marker for ccRCC patient. The results of in vitro assays indicated that knockdown of H19 reduced cell proliferation, invasion, and migration. Our data suggested that lncRNA H19 might be considered as a potential prognostic indicator and a target for gene therapy of ccRCC.

Key words: clear cell renal cell carcinoma, lncRNA H19, proliferation, metastasis

Renal cell carcinoma (RCC) is the most common urologic malignancy, accounting for approximately $3 \%$ of adult malignancies and causes about 90,000 deaths worldwide annually [1]. Clear cell renal cell carcinoma (ccRCC) is the largest subtype of RCC and accounts approximately $70 \%$ of these tumors [2]. ccRCC respond poorly to chemotherapy and radiotherapy and surgery remains the only curative treatment [3]. Early detection is of great importance for patient outcome, the 5-year survival for patients diagnosed with organ-confined disease is approximately $90 \%$, whereas the prognosis of patients with distant metastasis remains poor with a 5-year survival of less than 10\% [4,5]. Because of the poor prognosis associated with metastasis in RCC, it is necessary to identify new sensitive, reliable biomarkers enabling prediction of early metastasis and to develop new targeted therapies for RCC.
The rapid development of RNA genomics has highlighted the role of long non-coding RNAs (lncRNAs) in posttranscriptional regulation of human tumors [6]. LncRNAs are defined as endogenous cellular RNAs of more than 200 nucleotides in length and implicated in a myriad of molecular functions, such as modulation of alternative splicing, chromatin remodeling and RNA metabolism [7]. An increasing number of data show that lncRNAs play a key role in cancer development and metastasis [8]. For example, lncRNA HOTAIR in the HOX locus can increase the invasiveness and metastatic potential of human breast cancer by inducing genome-wide retargeting of Polycomb Repressive Complex 2 (PRC2) to an occupancy pattern, which leads to histone $\mathrm{H} 3$ lysine 27 trimethylation and changes in gene expression [9]. LncRNA MALAT-1 is increased in prostate cancer and higher MALAT-1 expression is correlated with gleason score, 
prostate specific antigen, tumor stage and castration resistant prostate cancer. Moreover down-regulation of MALAT-1 can inhibit prostate cancer cell growth, invasion and migration [10]. LncRNA SPRY4-IT1 is overexpressed in esophageal squamous cell carcinoma and is associated with clinical pathological stages and overall survival rate of the disease. Knockdown of SPRY4-IT1 reduced cell proliferation, invasiveness, and migration [11].

LncRNA H19 is a paternally imprinted gene located close to the telomeric region of chromosome $11 \mathrm{p} 15.5$, an area that is frequently involved in pediatric and adult tumors. While the understanding of expression and imprinting of lncRNA H19 has progressed in recent years, its function remains enigmatic. Recent studies showed the lncRNA H19 is overexpressed in several malignancies such as breast cancer, bladder cancer, glioma, and gastric cancer, and lncRNA H19 differential expression is linked to cancer metastasis. Berteaux et al. showed that a novel $\mathrm{H} 19$ antisense RNA overexpressed in breast cancer contributes to paternal IGF2 expression [12]. Luo et al. found that lncRNA H19 levels are remarkably increased in bladder cancer tissues, and upregulated $\mathrm{H} 19$ promotes bladder cancer cell migration in vitro and in vivo. Moreover they suggested that upregulated H19 enhances bladder cancer metastasis by associating with EZH2 and inhibiting E-cad expression [13]. Shi et al. indicated that in glioma H19 expression was significantly higher in high grade glioma tissues than that in low grade glioma tissues, and $\mathrm{H} 19$ regulated glioma cell invasion by deriving miR-675 and inhibited CDH13 [14]. Li et al. suggested that the expression of IncRNA H19 is increased in gastric cancer compared to that in the adjacent normal tissues and correlated with poor prognosis. Moreover overexpression of H19 promoted the features of gastric cancer including proliferation, migration, invasion and metastasis [15]. However, the lncRNA H19 expression in ccRCC and the underlying mechanism are still unclear.

In the current study, we explored the expression of IncRNA H19 in ccRCC and their relationship with clinicopathological factors. Furthermore, the roles of H19 in the proliferation, migration, and invasion were investigated in vitro to gain a better understanding of the function of lncRNA H19 in ccRCC. Our data revealed that lncRNA H19 is a novel molecule involved in the progression of ccRCC.

\section{Patients and methods}

Patients and specimens. A total of 92 ccRCC patients who underwent radical nephrectomy between 2007 and 2010 in our hospital (Zhengzhou Central Hospital Affiliated To Zhengzhou University) were enrolled in the study. All the specimens including cancer and paired normal tissues were placed in liquid nitrogen until use. 57 of these 92 patients were men and 35 were women. The median age of the patients was 58 years. Clinicopathological characteristics in our study are presented in Table1. All patients were followed up until September 2013. For the use of these clinical materials for research purposes, prior patient's consent and approval from the Institute Research Ethics Committee were obtained.

Cell culture. The human renal cancer cell lines 786-O, $\mathrm{ACHN}$, and Caki-1 were purchased from the Cell Bank of Type Culture Collection of Chinese Academy of Sciences (CCCAS, China). Nonmalignant renal cell line HK-2 was purchased from the American Type Culture Collection (ATCC, USA). HK-2 cells were cultured in KSFM medium (Gibco), and other cells were cultured in RPMI-1640 medium (Gibco) with 10\% fetal bovine serum (Gibco), $50 \mathrm{U} / \mathrm{ml}$ of penicillin and $50 \mu \mathrm{g} / \mathrm{ml}$ of streptomycin. All cells were cultured in a sterile incubator maintained at $37^{\circ} \mathrm{C}$ with $5 \% \mathrm{CO}_{2}$.

Cell transfection. The sequences of si-H19 were 5'-GCAGGACAUGACAUGGUCCdTdT-3'and si-NC were 5'-UUC UCCGAACGUGUCACGUUU-3'. Human renal cancer 786-O cells were transfected with either $50 \mathrm{nmol} \mathrm{si-H19}$ or si-NC using Lipofectamine 2000 transfection reagent (Life Technologies) according to the manufacturer's instruction. After 48 hours, cells transfected with siRNA were harvested for qRT-PCR to determine the transfection efficiency.

Cell proliferation assay. To determine cell growth, $786-\mathrm{O}$ cells were plated in a 24 -well plates $\left(5 \times 10^{4}\right.$ cells/ well) and transfected with siRNA. And cell proliferation was determined using a MTT (3-(4, 5-Dimethylthiazol-2-yl)-2, 5-diphenyltetrazolium bromide) according to the manufacturer's protocol. The absorbance intensity was measured using a microplate reader (Molecular Devices). Three independent experiments (three replicates in each) were performed.

Apoptosis assay. To determine cell apoptosis, 786-O cells were plated into 6 -well plates $\left(1 \times 10^{6}\right.$ cells/well $)$ in antibioticfree medium, after being transfected with siRNA. Cells for apoptosis analysis were collected and washed twice and stained with FITC-Annexin V and PI, using the FITC-Annexin $\mathrm{V}$ Apoptosis Detection Kit (BD Biosciences) according to the

Table 1. Clinical characteristics of the ccRCC patients

\begin{tabular}{|c|c|c|c|c|c|}
\hline \multirow[t]{2}{*}{ Parameters } & \multirow[t]{2}{*}{ Group } & \multirow[t]{2}{*}{ Total } & \multicolumn{2}{|c|}{ H19 expression } & \multirow{2}{*}{$\begin{array}{c}P \\
\text { value }\end{array}$} \\
\hline & & & High & Low & \\
\hline \multirow[t]{2}{*}{ Gender } & Male & 57 & 26 & 31 & \multirow[t]{2}{*}{0.993} \\
\hline & Female & 35 & 16 & 19 & \\
\hline \multirow[t]{2}{*}{ Age (years) } & $<60$ & 52 & 22 & 30 & \multirow[t]{2}{*}{0.463} \\
\hline & $\geq 60$ & 40 & 20 & 20 & \\
\hline \multirow[t]{2}{*}{ Tumor size $(\mathrm{cm})$} & $<4 \mathrm{~cm}$ & 48 & 26 & 22 & \multirow[t]{2}{*}{0.087} \\
\hline & $\geq 4 \mathrm{~cm}$ & 44 & 16 & 28 & \\
\hline \multirow[t]{2}{*}{ Histological grade } & G1-G2 & 56 & 15 & 41 & \multirow[t]{2}{*}{0.000} \\
\hline & G3-G4 & 36 & 27 & 9 & \\
\hline \multirow[t]{2}{*}{ Tumor stage } & $\mathrm{T} 1-\mathrm{T} 2$ & 55 & 18 & 37 & \multirow[t]{2}{*}{0.002} \\
\hline & $\mathrm{T} 3-\mathrm{T} 4$ & 37 & 24 & 13 & \\
\hline \multirow{2}{*}{$\begin{array}{l}\text { Lymph nodes } \\
\text { metastasis }\end{array}$} & Negative & 73 & 27 & 46 & \multirow[t]{2}{*}{0.001} \\
\hline & Positive & 19 & 15 & 4 & \\
\hline \multirow[t]{2}{*}{ Distant metastasis } & Negative & 81 & 33 & 48 & \multirow[t]{2}{*}{0.010} \\
\hline & Positive & 11 & 9 & 2 & \\
\hline
\end{tabular}


manufacturer's instructions. Apoptotic cells were analyzed by using flow cytometry (FACS, BD Bioscience).

Wound-healing assay. To determine cell migration, 786-O cells transfected with siRNA were seeded into 12-well plates and allowed to grow to $90-95 \%$ confluence. Before scratching, cells were starved for 24 hours in the medium with $1 \%$ FBS. Similar sized wounds were introduced to monolayer cells using a sterile white pipette tip. Wounded monolayer cells were washed three times by PBS to remove cell debris and then cultured. The speed of wound closure was monitored and photographed at $0 \mathrm{~d} 48$ hours.

Transwell invasion assays. To determine cell invasion capacity, Matrigel-coated invasion chambers ( $8 \mu \mathrm{m}$; Costar) were used according to the manufacturer's protocol. Briefly, siRNA transfected cells were harvested, resuspended $\left(1 \times 10^{5}\right.$ cells/well) in $200 \mu \mathrm{L}$ serum-free medium, and transferred to the upper chamber of the Matrigel-coated inserts, culture medium containing $10 \%$ FBS was placed in the bottom chamber. The cells were incubated for 24 hours at $37^{\circ} \mathrm{C}$. After incubation, the non-invaded cells on the upper membrane surface were removed with a cotton tip, and the cells that passed through the filter were fixed and stained using $0.1 \%$ crystal violet. Numbers of invaded cells were counted in 5 randomly selected high power fields under a microscope.

RNA extraction and quantitative real-time PCR. Total RNA was extracted from tissues or cells using the Trizol reagent (Invitrogen) according to the manufacturer's instructions. Total RNA was eluted with RNase-free water and stored at $-80^{\circ} \mathrm{C}$. RNA concentrations were determined by Epoch spectrophotometry.

The quantitative Real-time PCR (qRT-PCR) was performed by using SYBR Green PCR Master Mix in a Fast Real-time PCR 7900 System (Applied Biosystems). The gene-specific primers were as follows: H19 (forward: 5'-ATCGGTGCCTCAGCGTTCGG-3'; reverse: 5'-CTGTCCTCGCCGTCACACCG-3'). U6 snRNA (forward: 5'-GGGCAGGAAGAGGGCCTAT-3'; reverse: 5'-TATGGCTAGCATGACTGGT-3') was amplified in parallel as the internal control. PCR reactions were performed at $50^{\circ} \mathrm{C}$ for $2 \mathrm{~min}$, followed by 40 cycles of $95^{\circ} \mathrm{C}$ for $15 \mathrm{~s}$ and $60^{\circ} \mathrm{C}$ for $1 \mathrm{~min}$. Data was collected and analyzed by SDS2.3 Software (Applied Biosystems). The relative quantitative value was expressed by the $2^{-\Delta \Delta \mathrm{Ct}}$ method. Each experiment was performed in triplicates and repeated three times.

Statistical analysis. All statistical analyses were performed using SPSS version 18.0 software. Data between groups in cell experiments were analyzed by one-way analysis of variance (ANOVA). Categorical data were analyzed using the twoside chi-square test. Overall survival was estimated by using Kaplan-Meier method, and univariate analysis was conducted by log-rank test. The Cox proportional hazards model was used in the multivariate analysis. Values of $\mathrm{P}<0.05$ were considered statistically significant.

\section{Results}

LncRNA H19 is up-regulated in renal cancer. To assess the role of H19 in renal cancer progression, we first examined the H19 expression levels in renal cancer tissues using qRT-PCR. We verified that $\mathrm{H} 19$ expression was significantly upregulated in clinical ccRCC tissues compared to adjacent normal tissues $(\mathrm{P}<0.05$, Fig. $1 \mathrm{~A})$. Expression was also examined by qRT-PCR in 3 renal cancer cell lines and nonmalignant renal cell line HK-2. Our data showed that H19 expression was higher in renal cancer cell lines than that in HK-2 cells $(\mathrm{P}<0.05$, Fig. $1 \mathrm{C})$.

Then, patients with ccRCC were divided into two groups based on the fold-change of H19 expression as follows: H19-

Table 2. Univariate and multivariate analysis of factors influencing survival in patients with ccRCC

\begin{tabular}{|c|c|c|c|c|c|c|}
\hline \multirow[t]{2}{*}{ Variable } & \multicolumn{3}{|c|}{ Univariate analysis } & \multicolumn{3}{|c|}{ Multivariate analysis } \\
\hline & Risk ratio & $95 \%$ CI & $P$-value & Risk ratio & $95 \%$ CI & $P$-value \\
\hline Gender & 1.414 & $0.615-2.183$ & 0.279 & & & \\
\hline \multicolumn{7}{|l|}{ Male vs Female } \\
\hline Age (years) & 1.971 & $0.432-2.718$ & 0.197 & & & \\
\hline \multicolumn{7}{|l|}{$\geq 60$ vs $<60$} \\
\hline Tumor size & 2.462 & $0.972-3.919$ & 0.218 & & & \\
\hline \multicolumn{7}{|l|}{$\geq 4 \mathrm{~cm}$ vs $<4 \mathrm{~cm}$} \\
\hline Histological grade & 3.271 & $1.735-5.647$ & 0.008 & 3.114 & $1.407-5.006$ & 0.013 \\
\hline \multicolumn{7}{|l|}{ G3-G4 vs G1-G2 } \\
\hline Tumor stage & 2.976 & $1.912-5.917$ & 0.017 & 2.514 & $1.417-5.247$ & 0.023 \\
\hline \multicolumn{7}{|l|}{ T3-T4 vs T1-T2 } \\
\hline Lymph node & 4.783 & $2.194-9.266$ & 0.008 & 4.117 & $1.911-7.378$ & 0.013 \\
\hline \multicolumn{7}{|l|}{ Positive vs Negative } \\
\hline Distant metastasis & 5.529 & $2.197-11.224$ & 0.004 & 4.873 & $2.339-9.528$ & 0.002 \\
\hline \multicolumn{7}{|l|}{ Positive vs Negative } \\
\hline $\begin{array}{l}\text { H19 } \\
\text { high vs low }\end{array}$ & 4.613 & $1.732-8.1974$ & 0.005 & 3.894 & $1.872-8.014$ & $<0.001$ \\
\hline
\end{tabular}




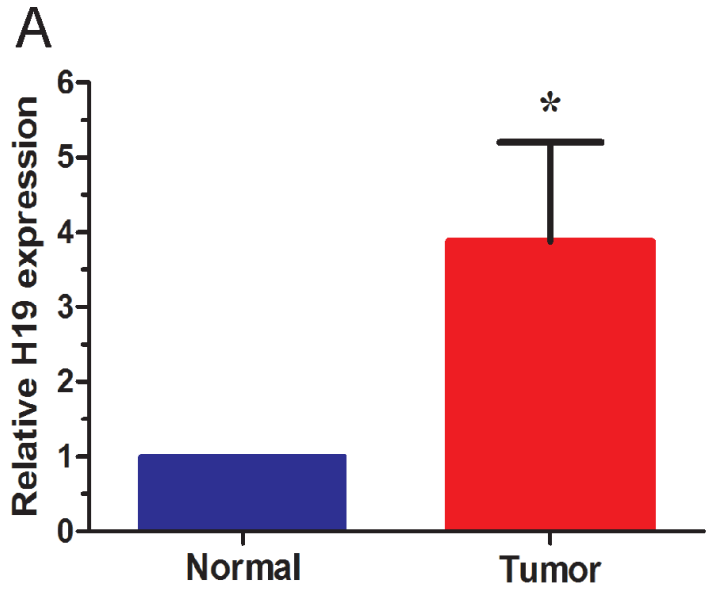

B


Figure 1. IncRNA H19 levels were upregulated in ccRCC tissues and renal cancer cells. The relative $\mathrm{H} 19$ expression levels were determined using qRT-PCR and demonstrated using the comparative $\Delta \mathrm{Ct}$ method. A. Higher relative $\mathrm{H} 19$ levels were detected in ccRCC tissues than in adjacent normal tissues. B. LncRNA H19 expression was classified into two groups. C. Higher expression levels of $\mathrm{H} 19$ were detected in 3 renal cancer cell lines than HK-2 cell line. Results are expressed as mean \pm SD for three replicate determination. ${ }^{*} \mathbf{P}<\mathbf{0 . 0 5}$.



Figure 2. The survival analysis of H19. Patients with higher H19 expression in ccRCC tissues were closely correlated with poorer overall survival than patients with lower $\mathrm{H} 19$ expression $(\mathrm{P}<0.05)$.

high ( $\geq 3.8$-fold, $n=42$ ) and H19-low ( $<3.8$-fold, $n=50$ )(Fig.1B). As shown in Table 1, H19 upregulation was correlated with tumor stage, lymph node metastasis, and distance metastasis $(\mathrm{P}<0.05)$, but not correlated with patient's age, gender, histological grade, and tumor size $(\mathrm{P}>0.05)$. Taken together, these data suggested that increased $\mathrm{H} 19$ expression was associated with the progression and development of ccRCC.

High expression of $\mathrm{H} 19$ predicted worse prognosis in ccRCC patients. Kaplan-Meier analysis showed that patients with high expression of $\mathrm{H} 19$ were associated with shorter overall survival compared to those with low expression of H19 (log-rank test, $\mathrm{P}<0.05$ ) (Fig. 2). As shown in Table 2, H19 expression level, tumor stage, lymph node metastasis, and distance metastasis were significantly correlated with overall survival rate of patients with ccRCC $(\mathrm{P}<0.05)$. A multivariate analysis showed that relative expression of $\mathrm{H} 19$, tumor stage, lymph node metastasis, and distance metastasis were independent prognostic factors for the overall survival of ccRCC patients (Table 2). These data indicated that H19 expression is a potential independent prognostic factor in ccRCC patients.

Knockdown of $\mathrm{H} 19$ decreased renal cancer cell proliferation and enhanced cell apoptosis. To study the biological role of H19 in cell growth, renal cancer cell lines transfected with si-H19 were analyzed. LncRNA H19 levels were significantly decreased in 786-O cells transfected with si-H19 (Fig. 3A). Downregulation of $\mathrm{H} 19$ significantly decreased the growth of 786-O cells (Fig. 3B). We also assessed whether downregulated expression of $\mathrm{H} 19$ contributed to cell apoptosis. Fig.3C showed that downregulation of $\mathrm{H} 19$ expression promoted 786-O cell apoptosis. These data suggested that downregulation of H19 has an inhibitory effect on growth of renal cancer cells. 
Knockdown of $\mathrm{H} 19$ inhibits renal cancer cell migration and invasion in vitro. We further assessed the effects of $\mathrm{H} 19$ on cell migration and invasion, which are key determinants of malignant progression and metastasis. Wound healing assay showed that 786-O cells transfected with si-H19 have a slower healing capacity than that in si-NC group $(\mathrm{P}<0.05$, Fig. 3D $)$.

Transwell invasion assays were under taken to assess the effect of $\mathrm{H} 19$ on the invasiveness of renal cancer cells. The results
A



B

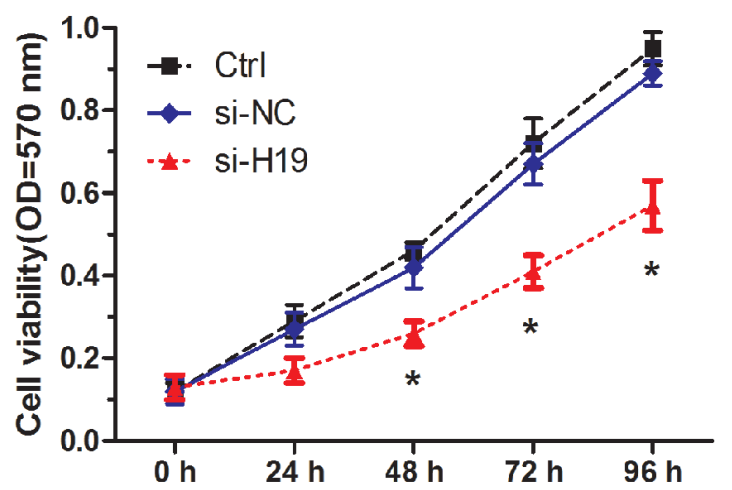

C

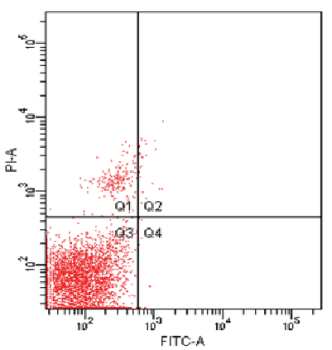

Ctrl

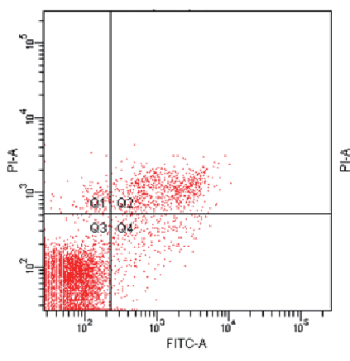

si-NC

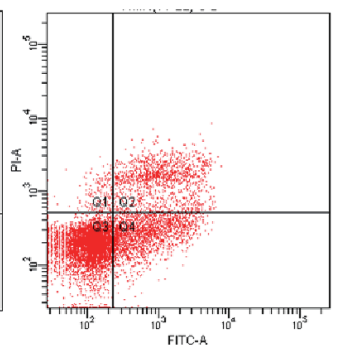

si-H19

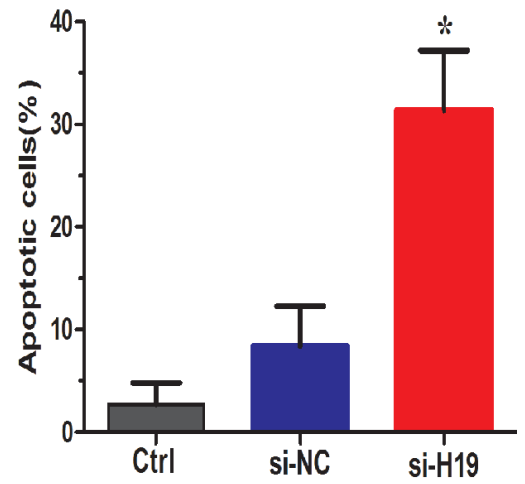

$E$
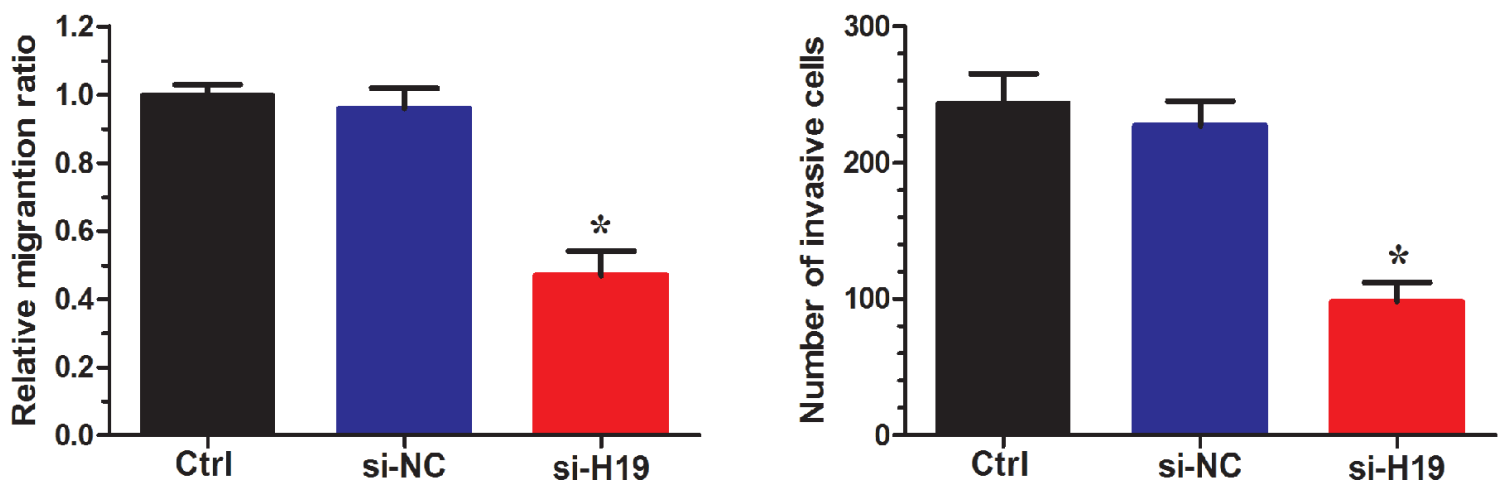

Figure 3. Evaluation of biological functions of $\mathrm{H} 19$ in renal cancer cells. A. Transfection efficiency of si-H19 in renal cancer cells was indicated by qRT-PCR. B. si-H19 inhibited the proliferation of renal cancer cells. 786-O cells were plated in a 24 -well plate and transfected with si-H19, and cell proliferation was determined at the indicated time points using a MTT assay. C. si-H19 promoted apoptosis of renal cancer cells. D. si-H19 inhibited the migration of renal cancer cells. Wound healing assay was performed to investigate the effects of si-H19 downregulation on cell migration in $786-\mathrm{O}$ cells. E. si-H19 inhibited the invasiveness of renal cancer cells. Transwell invasion assay was performed to investigate the effects of si-H19 downregulation on cell invasion in 786-O cells. Ctrl, cells without transfection; si-NC, cells transfected with nonspecific siRNA; si-H19, cells transfected with H19-specific siRNA. Results are expressed as means \pm SD for three replicate determination. ${ }^{\star} \mathbf{P}<0.05$. 
indicated that the invasion ability of si-H19 infected 786-O cells was reduced compared with the si-NC group $(\mathrm{P}<0.05$, Fig. 3E ). These results suggested a functional role for $\mathrm{H} 19$ in mediating cell migration and invasion in renal cancer.

\section{Discussion}

Renal cell carcinoma remains to be one of the highest causes of cancer mortality worldwide. So finding new molecular targets for its diagnosis, prognosis and treatment have the potential to improve the clinical strategies and outcomes of this disease. With the development of whole genome sequencing technology, it was determined that less than $2 \%$ of the mammalian genome is in protein encoded regions and the remainder is in non coding RNAs [16]. Among them are long non-coding RNAs (lncRNAs), which are more than 200 nucleotides in length and unable to be translated into proteins [7]. Recently, many studies showed that lncRNAs play key roles in tumor development and can be used to develop as biomarkers and prognosis factors [17,18]. IncRNA H19, which lies within $200 \mathrm{k}$ bp downstream of the IGF2 gene, expressed from the maternal allele in several tissues during embryo development [19]. Recent studies showed H19 gene was reexpressed and characterized as a tumor oncogenic factor in several cancer tissues such as breast cancer, bladder cancer, glioma, and gastric cancer [12-15]. However, other studies suggested a potential tumor suppressor effect of H19, such as Wilms' tumors and embryonic rhabdomyosarcoma $[20,21]$. Here, we want to explore possible roles of H19 played in ccRCC.

In this study, we found the clinical significance of H19 in ccRCC patients for the first time. By using qRT-PCR, our data showed that lncRNA H19 was significantly increased in ccRCC tissues and renal cancer cell lines to a greater extent than in adjacent normal tissues and nonmalignant renal cell line HK-2. Furthermore, we found that H19 expression was correlated with tumor stage, lymph node metastasis, and distance metastasis, but no correlation with patient's age, gender, histological grade, and tumor size was observed. In addition, patients with high H19 expression had a poorer prognosis than those with low H19 expression and lcnRNA H19 could be an independent prognostic factor in patients with ccRCC. These results suggested that lcnRNA H19 play important roles in development, tumorigenesis, and progression of renal cancer.

Ma's study indicated that $\mathrm{H} 19$ promotes pancreatic cancer metastasis by derepressing let-7's suppression on its target HMGA2-mediated EMTH19. siRNA-mediated H19 knockdown in human pancreatic cancer cells result in defects in cell migration, and invasion in vitro [22]. Li et al. found that overexpression of H19 in gastric cancer cells promotes cell proliferation, migration and invasion in vitro. In addition, overexpression of $\mathrm{H} 19$ promotes tumorigenesis and metastasis in vivo [15]. From our clinical pathological data, we found that high H19 expression is closely associated with tumor stage, lymph node metastasis, and distance metastasis, thus we sup- posed that $\mathrm{H} 19$ may also regulate the growth and metastasis of renal cancer. It is necessary to identify the biological function of H19 in renal cancer cells.

To further understand the mechanism of H19 in renal cancer cell process, in vitro experiments were conducted. siRNA-mediated knockdown of H19 significantly decreased the growth and metastasis capability of 786-O cells compared with control group, which suggested that IncRNA H19 play an important role in the development of renal cancer.

Taken together, Our study for the first time found that lncRNA H19 was upregulated in ccRCC tissues and correlation with tumor stage, lymph node metastasis, and distant metastasis. Furthermore, H19 expression was suggested to be an independent marker for predicting the clinical outcome of patients with ccRCC. In addition, knockdown the expression of H19 can significantly decrease the growth and metastatic capacity of renal cancer cells. Our results indicated that H19 could be a promising biomarker and a therapeutic target for renal cancer.

\section{References}

[1] JEMAL A, BRAY F, CENTER MM, FERLAY J, WARD E. et al. Global cancer statistics. CA Cancer J Clin 2011; 61: 69-90. http://dx.doi.org/10.3322/caac.20107

[2] RINI BI, CAMPBELL SC, ESCUDIER B Renal cell carcinoma. Lancet 2009; 373: 1119-1132. http://dx.doi.org/10.1016/ S0140-6736(09)60229-4

[3] YANG F, SHAO Y, YANG F, LIU M, HUANG J. et al. Valproic acid upregulates NKG2D ligand expression and enhances susceptibility of human renal carcinoma cells to NK cellmediated cytotoxicity. Arch Med Sci 2013; 9: 323-31. http:// dx.doi.org/10.5114/aoms.2013.34413

[4] LJUNGBERG B, COWAN NC, HANBURY DC, HORA M, KUCZYK MA. et al. EAU guidelines on renal cell carcinoma: the 2010 update. Eur Urol 2010; 58: 398-406. http://dx.doi. org/10.1016/j.eururo.2010.06.032

[5] MOTZER RJ, ESCUDIER B, BUKOWSKI R, RINI BI, HUTSON TE. et al. Prognostic factors for survival in 1059 patients treated with sunitinib for metastatic renal cell carcinoma. Brit J Cancer 2013; 108: 2470-2477. http://dx.doi.org/10.1038/ bjc. 2013.236

[6] ESTELLER M Non-coding RNAs in human disease. Nat Rev Genet 2011; 12(12): 861-874. http://dx.doi.org/10.1038/ $\underline{\operatorname{nrg} 3074}$

[7] SPIZZO R, ALMEIDA MI, COLOMBATTI A, CALIN GA Long non-coding RNAs and cancer: a new frontier of translational research\&quest. Oncogene 2012; 31: 4577-4587. http:// dx.doi.org/10.1038/onc.2011.621

[8] GIBB EA, BROWN CJ, LAM WL The functional role of long non-coding RNA in human carcinomas. Mol Cancer 2011; 10: 38-55. http://dx.doi.org/10.1186/1476-4598-10-38

[9] GUPTA RA, SHAH N, WANG KC, KIM J, HORLINGS HM. et al. Long non-coding RNA HOTAIR reprograms chromatin state to promote cancer metastasis. Nature 2010; 464: 1071-1076. http://dx.doi.org/10.1038/nature08975 
[10] REN S, LIU Y, XU W, SUN Y, LU J. et al. Long noncoding RNA MALAT- 1 is a new potential therapeutic target for castration resistant prostate cancer. J Urology 2013; 190: 2278-2287. http://dx.doi.org/10.1016/j.juro.2013.07.001

[11] XIE HW, WU QQ, ZHU B, CHEN FJ, JI L. et al. Long noncoding RNA SPRY4-IT1 is upregulated in esophageal squamous cell carcinoma and associated with poor prognosis. Tumour Biol 2014; 1-12.

[12] BERTEAUX N, APTEL N, CATHALA G, GENTON C, COLL J. et al. A novel H19 antisense RNA overexpressed in breast cancer contributes to paternal IGF2 expression. Mol Cell Biol 2008; 28: 6731-6745. http://dx.doi.org/10.1128/MCB.02103$\underline{07}$

[13] LUO M, LI Z, WANG W, ZENG Y, LIU Z. et al. Long noncoding RNA H19 increases bladder cancer metastasis by associating with EZH2 and inhibiting E-cadherin expression. Cancer Lett 2013; 333: 213-221. http://dx.doi.org/10.1016/j. canlet.2013.01.033

[14] SHI Y, WANG Y, LUAN W, WANG P, TAO T. et al. Long noncoding RNA H19 promotes glioma cell Invasion by deriving miR-675. PloS one 2014; 9: e86295. http://dx.doi.org/10.1371/ journal.pone.0086295

[15] LI H, YU B, LI J, SU L, YAN M. et al. Overexpression of lncRNA H19 enhances carcinogenesis and metastasis of gastric cancer. Oncotarget 2014; 5: 2318-2329.
[16] ZHANG EB, KONG R, YIN DD, YOU LH, SUN M. et al. Long noncoding RNA ANRIL indicates a poor prognosis of gastric cancer and promotes tumor growth by epigenetically silencing of miR-99a/miR-449a. Oncotarget 2014; 5: 2276-2292.

[17] AMARAL PP, MATTICK JS NONCODING RNA IN DEVELOPMENT. Mamm Genome 2008; 19(7-8): 454-492. http://dx.doi.org/10.1007/s00335-008-9136-7

[18] PRENSNER JR, CHINNAIYAN AM The emergence of lncRNAs in cancer biology. Cancer Discov 2011; 1: 391-407. http://dx.doi.org/10.1158/2159-8290.CD-11-0209

[19] KAFFER CR, SRIVASTAVA M, PARK KY, IVES E, HSIEH S. et al. A transcriptional insulator at the imprinted H19/Igf2 locus. Genes Dev 2000; 14: 1908-1919.

[20] MOULTON T, CRENSHAW T, HAO Y, MOOSIKASUWAN J, LIN N. et al. Epigenetic lesions at the H19 locus in Wilms tumour patients. Nat Genet 1994; 7: 440-447. http://dx.doi. org/10.1038/ng0794-440

[21] CASOLA S, PEDONE PV, CAVAZZANA AO, BASSO G, LUKSCH R. et al. Expression and parental imprinting of the H19 gene in human rhabdomyosarcoma. Oncogene 1997; 14: 1503-1510. http://dx.doi.org/10.1038/sj.onc.1200956

[22] MA C, NONG K, ZHU H, WANG W, HUANG X. et al. H19 promotes pancreatic cancer metastasis by derepressing let-7's suppression on its target HMGA2-mediated EMT. Tumour Biol 2014; 1-7. 